\title{
NeuroImage
}

ELSEVIER

\section{Human cortical EEG rhythms during long-term episodic memory task. A high-resolution EEG study of the HERA model}

\author{
Claudio Babiloni, ${ }^{\text {a,b,c }}$ Fabio Babiloni, ${ }^{\text {a, } *}$ Filippo Carducci, ${ }^{\text {a,b,c }}$ Stefano Cappa, ${ }^{\mathrm{d}}$ Febo Cincotti, ${ }^{\text {a }}$ \\ Claudio Del Percio, ${ }^{\mathrm{a}}$ Carlo Miniussi, ${ }^{\mathrm{b}}$ Davide Vito Moretti, ${ }^{\mathrm{a}}$ Patrizio Pasqualetti,, $\mathrm{c}$ \\ Simone Rossi, ${ }^{\mathrm{e}}$ Katiuscia Sosta, ${ }^{\mathrm{b}}$ and Paolo Maria Rossini ${ }^{\mathrm{b}, \mathrm{c}, \mathrm{f}}$ \\ ${ }^{a}$ Dipartimento di Fisiologia Umana e Farmacologia Sezione di EEG ad Alta Risoluzione Università degli Studi di Roma "La Sapienza" P.le Aldo Moro 5 \\ 00185 Rome, Italy \\ ${ }^{\mathrm{b}}$ IRCCS S Giovanni di Dio, fatebenefratelli, Brescia, Italy \\ ${ }^{\mathrm{c}}$ AFaR.-Dip. di Neuroscienze, S. Giovanni Calibita, Fatebenefratelli Isola Tiberina, Rome, Italy \\ ${ }^{\mathrm{d}}$ Centro di Neuroscienze Cognitive, Università Salute-Vita S. Raffaele, Milan, Italy \\ ${ }^{\mathrm{e}}$ Dipartimento di Neuroscienze, Sezione Neurologia, Università di Siena, Siena, Italy \\ ${ }^{\mathrm{f}}$ Neurologia, Università Campus Biomedico, Rome, Italy
}

Received 12 August 2003; revised 17 November 2003; accepted 20 November 2003

\begin{abstract}
Many recent neuroimaging studies of episodic memory have indicated an asymmetry in prefrontal involvement, with the left prefrontal cortex more involved than the right in encoding, the right more than the left in retrieval (hemispheric encoding and retrieval asymmetry, or HERA model). In this electroencephalographic (EEG) high-resolution study, we studied brain rhythmicity during a visual episodic memory (recognition) task. The theta $(4-6 \mathrm{~Hz})$, alpha $(6-12 \mathrm{~Hz})$ and gamma $(28-48 \mathrm{~Hz})$ oscillations were investigated during a visuospatial longterm episodic memory task including an encoding (ENC) and retrieval (RET) phases. During the ENC phase, 25 figures representing interiors of buildings ("indoor") were randomly intermingled with 25 figures representing landscapes ("landscapes"). Subject's response was given at left ("indoor") or right ("landscapes") mouse button. During the RET phase (1 h later), 25 figures representing previously presented "indoor" pictures ("tests") were randomly intermingled with 25 figures representing novel "indoor" ("distractors"). Again, a mouse response was required. Theta and alpha EEG results showed no change of frontal rhythmicity. In contrast, the HERA prediction of asymmetry was fitted only by EEG gamma responses, but only in the posterior parietal areas. The ENC phase was associated with gamma EEG oscillations over left parietal cortex. Afterward, the RET phase was associated with gamma EEG oscillations predominantly over right parietal cortex. The predicted HERA asymmetry was thus observed in an unexpected location. This discrepancy may be due to the differential sensitivity of neuroimaging methods to selected components of cognitive processing. The strict relation between gamma response and perception suggests that retrieval processes of long-term
\end{abstract}

\footnotetext{
* Corresponding author. Fax: +39-06-49910917.

E-mail address: Claudio.babiloni@uniroma1.it (F. Babiloni).

Available online on ScienceDirect (www.sciencedirect.com.)
}

memory deeply impinged upon sensory representation of the stored material.

(c) 2004 Elsevier Inc. All rights reserved.

Keywords: Long-term episodic memory; Encoding; Retrieval; Electroencephalography (EEG); Event-related desynchronization/synchronization (ERD/ERS); Theta rhythm; Alpha rhythm; Gamma rhythm; Hemispherical encoding retrieval asymmetry (HERA)

\section{Introduction}

Positron emission tomography (PET), functional magnetic resonance imaging (fMRI) and event-related potential (ERP) studies have shown that, in healthy young individuals, long-term episodic memory is associated with "activation" of discrete frontal neuronal networks (Brewer et al., 1998; Buckner and Wheeler, 2000; Fletcher and Henson, 2001; Kirchhoff et al., 2000). In particular, different aspects of the memory performance are represented in right and left prefrontal areas (Fletcher and Henson, 2001), which cooperate with medial temporal lobe structures for the retrieval of both verbal and nonverbal information (Buckner and Wheeler, 2000). For example, a focal "activation" of right prefrontal cortex and bilateral mediotemporal regions (i.e., parahippocampal) during the encoding phase predicts a successful recognition of visual stimuli in a later retrieval phase (Brewer et al., 1998).

Also, the brain rhythmicity of electroencephalographic (EEG) oscillations (i.e., event-related desynchronization/synchronization, or ERD/ERS) can be a valid methodology to investigate long-term memory tasks. It has been suggested that: (i) the desynchronization at the upper alpha band $(10-12 \mathrm{~Hz})$ is related to semantic longterm memory processes (Dujardin et al., 1994, 1995; Klimesch et al., 1996, 1997, 1999), (ii) the search and retrieval processes in 
(semantic) long-term memory are reflected by upper alpha oscillations in thalamocortical feedback loops and (iii) the encoding of new information is reflected by theta oscillations in hippocampocortical feedback loops (Dujardin et al., 1994, 1995; Klimesch et al., 1996, 1997, 1999).

Complementary to neuroimaging (fMRI, PET), ERP and ERD/ERS techniques, repetitive transcranial magnetic stimulation (rTMS) indexes the causal role of a given cortical area for a certain task. Indeed, rTMS temporarily interferes with local neural processes and can cause a transient decline of behavioral performance if the stimulated area plays a relevant functional role in the task considered (Hallett, 2000; Pascual-Leone et al., 2000; Rossini and Rossi, 1998; Walsh and Cowey, 2000). The rTMS approach has recently confirmed a functional inter-hemispherical asymmetry of dorsolateral prefrontal cortex (DLPFC) in healthy young individuals involved in long-term memory challenges, both in verbal (Sandrini et al., 2004) and visuospatial domains (Rossi et al., 2001). For the latter, the rTMS of left DLPFC temporarily disabled the encoding of complex pictures (i.e., the same material used for the current study), while the rTMS of right DLPFC mainly impaired the retrieval of the encoded visual information. These results agreed with the so-called hemispherical encoding retrieval asymmetry (HERA) theory on the functional inter-hemispherical asymmetry during the long-term memorization of episodes (Lepage et al., 2000; Nyberg et al., 1996; Tulving et al., 1994). This model is now the focus of a lively discussion based on an increasing number of imaging studies that have attempted to characterize other factors affecting both the hemispheric asymmetry and the functional neuroanatomical subdivisions of frontal activation (Habib et al., 2003). It should be remarked that a HERA pattern is not an absolute feature of cortical activity during long-term memorization. Rather, the level or existence of HERA is affected and in some cases abolished by the nature of the material to be memorized (level of detail, difficulty, familiarity, verbal content, etc.) as shown by recent studies (Brewer et al., 1998; Buckner et al., 1999; Nolde et al., 1998; Ranganath et al., 2000; Sandrini et al., 2004; Wagner et al., 1999).

In this high-resolution EEG study, the theta (4-6 Hz), alpha (6$12 \mathrm{~Hz})$ and gamma $(28-48 \mathrm{~Hz})$ oscillations were investigated during a visuospatial long-term episodic memory task that was affected by rTMS at prefrontal areas according to the HERA model (Rossi et al., 2001). With respect to the mentioned rTMS investigation (Rossi et al., 2001), the present EEG approach allowed evaluation of the main brain rhythms of both frontal and parietal areas during the ENC and RET phases. The working hypothesis was that the brain rhythmicity represent a neural substrate of the hemispherical functional asymmetry as predicted by the HERA model.

\section{Materials and methods}

\section{Subjects}

Experiments were performed in 12 healthy, right-handed (Edinburgh Inventory, $91 \pm 2.5 \%$ ) adult volunteers (six males and six females, mean age 33 years $\pm 1.3 \mathrm{SE}$ ) who gave their informed written consent according to the declaration of Helsinki. The local institutional ethics committee approved general procedures.

\section{Experimental tasks}

Subjects were seated in a comfortable reclining armchair, placed in a dimly lit, sound-damped and electrically shielded room. They kept their forearms resting on armchairs, with right index finger resting between two buttons spaced $6 \mathrm{~cm}$ from each other. A computer monitor was placed in front of them (about $100 \mathrm{~cm}$ ). The experiment (Fig. 1) included an encoding (ENC) and a retrieval (RET) phase in which 50 complex colored magazine pictures were shown after a red central target given as a visual warning stimulus (presentation time of $1 \mathrm{~s}$ ). In the ENC condition, 25 figures representing interiors of apartments ("indoor") were randomly intermingled with 25 figures representing landscapes ("landscapes"). The figures were shown one-by-one (cue period, presentation time of $5 \mathrm{~s}$ ). Subjects were instructed to press with right index finger one of the two buttons (left = "indoor"; right = "landscapes") as quickly as possible after the appearance of a green central target at the center of the figure (go stimulus, presentation time of $1 \mathrm{~s}$ ). Remarkably, no mention to a "retrieval" phase was made before the encoding phase, according to a standard paradigm of "incidental memory". About $1 \mathrm{~h}$ later, the RET condition started. In this phase, 25 figures representing previously presented "indoor" ("tests") were randomly intermingled with 25 figures representing novel "indoor" ("distractors"). Subjects were asked to discriminate between "tests" and "distractors" by pressing with right index finger one of the two buttons (left = "tests"; right $=$ "distractors") immediately after the go stimulus.

The timing of warning stimulus, cue stimulus (picture presentation) and go stimulus was as in the "encoding" phase.

Before the ENC phase, a training of about 10 min was performed with a different set of figures to made familiar subjects with the experimental apparatus and general procedure used for the ENC-RET tasks, namely, to press the mouse buttons with the right index finger after visual stimuli.

\section{EEG recordings}

EEG data were recorded $(0.1-60 \mathrm{~Hz}$ bandpass; $256 \mathrm{~Hz}$ sampling frequency) with a 46-tin electrode cap referenced to linked ears. The electrodes were disposed according to an augmented 10-20 system (Table 1) and electrode impedance was kept lower than $5 \mathrm{k} \Omega$. The positions of the electrodes and landmarks were digitized.

Table 1

List including 46 electrodes (augmented 10-20 system) used for the present experiments

\begin{tabular}{l}
\hline Electrodes \\
\hline Fp1, Fp2 \\
AF7, AFz, AF8 \\
F7, F5, F3, F1, Fz, F2, F4, F6, F8 \\
FC3, FCZ, FC4 \\
T7, C5, C3, C1, Cz, C2, C4, C6, T8 \\
TP7, CP3, CPz, CP4, TP8 \\
P7, P5, P3, P1, Pz, P2, P4, P6, P8 \\
PO7, POz, PO8 \\
O1, Oz, O2
\end{tabular}

The position of the labels in the list roughly represents the position of the corresponding electrodes over the scalp. 
Electrooculogram $(0.1-60 \mathrm{~Hz}$ bandpass; $256 \mathrm{~Hz}$ sampling frequency) and surface electromyographic activity of bilateral extensor digitorum muscles (1-60 Hz bandpass; $256 \mathrm{~Hz}$ sampling frequency) were also collected. Electrooculogram monitored blinking or eye movements, whereas electromyogram monitored voluntary movements required by the task as well as involuntary mirror movements or other unspecific muscle activations.

\section{Off-line preliminary data analysis}

The EEG data were segmented in single trials each spanning from $-2.5 \mathrm{~s}$ to $+7.5 \mathrm{~s}$, the zero time being the onset of the cue stimulus (complex colored magazine picture). The EEG epochs were automatically and manually rejected. The computerized automatic procedure was based by a software package that included automatic procedures for (i) EOG artifact detection and correction, (ii) EMG analysis, (iii) EEG artifact analysis and (iv) optimization of the ratio between artifact-free EEG channels and trials to be rejected. The reject criteria were based on a threshold method and on a statistical method evaluating the non-stationarity of the EEG signal (Moretti et al., 2003). Then, two expert electroencephalographists manually confirmed the automatic selections.

Spatial resolution of artifact-free EEG data was enhanced by surface Laplacian estimation (regularized 3-D spline function; Babiloni et al., 1996, 1998). The surface Laplacian estimation acts as a spatial filter that reduces head volume conductor effects and eliminates electrode reference influence (Babiloni et al., 1996; Nunez, 1995). In some cases, the Laplacian values at the border electrodes were zeroed due to the unreliability of the spline Laplacian estimate at these electrodes.

The single-trial analysis was carefully repeated on the Laplacian-transformed EEG data to discard the single trials contaminated by residual computational artifacts. In 2 out of 12 subjects, the number of artifact-free EEG single trials was insufficient (i.e., lower than $30 \%$ of the individual data set). Consequently, the EEG data of 10 subjects were further considered for the final data analysis. The mean of the artifact-free EEG single trials was of 29 $( \pm 3 \mathrm{SE})$ for the ENC task and of $26( \pm 3 \mathrm{SE})$ for the RET task [no statistical difference; $F(1,9)=1.99 ; P=0.2]$. Concerning the RET task, the EEG single trials related to both previously presented ("tests") and novel ("distractors") pictures were averaged together to increase the signal-to-noise ratio. Indeed, there was an insufficient number of artifact-free EEG trials for each task for a separate data analysis.

Motor reaction time after the go stimulus was computed for all artifact-free EEG single trials.

\section{Determination of individual theta and alpha sub-bands}

Frequency bands of interest were theta, alpha 1, alpha 2, alpha 3 and gamma. Theta and alpha band limits were computed according to a standard procedure based on the peak of individual alpha frequency (IAF; Klimesch, 1996, 1999; Klimesch et al., 1998). The IAF was defined as the frequency showing maximum power density peak within extended alpha range $(6-14 \mathrm{~Hz})$. It was calculated during the baseline period (1-s epoch preceding the onset of the warning stimulus, -2000 to $-1000 \mathrm{~ms}$ ). With respect to the IAF, the frequencies of interest were (i) theta as IAF $-6 \mathrm{~Hz}$ to IAF $-4 \mathrm{~Hz}$, (ii) alpha 1 as the IAF $-4 \mathrm{~Hz}$ to IAF -2 $\mathrm{Hz}$, (iii) alpha 2 as IAF $-2 \mathrm{~Hz}$ to IAF, and (iv) alpha 3 as IAF to
$\mathrm{IAF}+2 \mathrm{~Hz}$ (Fig. 2). The power spectrum analysis was based on a FFT approach using Welch technique and Hanning windowing function.

The individual gamma frequency synchronization was defined as the spectral maximum of the ratio between baseline and cue period in the range of $28-48 \mathrm{~Hz}$. In 1 of 10 subjects, the noise did not allow the analysis of the gamma band. Consequently, 10 subjects were considered in the data analysis of theta and alpha rhythmicity, whereas 9 subjects were considered for the data analysis of the gamma rhythmicity.

\section{Computation of event-related desynchronization/synchronization (ERD/ERS)}

To quantify the event-related changes of EEG power, a standardized procedure was used, namely, the computation of event-related desynchronization/synchronization (ERD/ERS; Pfurtscheller and Aranibar, 1979; Pfurtscheller and Neuper, 1994; Pfurtscheller et al., 1997). The ERD/ERS of the individual theta, alpha 1, alpha 2, alpha 3 and gamma bands was computed as follows: Laplacian EEG time series were bandpassed (Bartlett function), squared, averaged across $120-\mathrm{ms}$ periods (to eight samples per second) and averaged across all single trials. The ERD/ERS was defined as the percentage decrement/increment of instant power density at the "event" compared to a "pre-event" baseline (from -2 to $-1 \mathrm{~s}$ ).

A spline interpolating function (Babiloni et al., 1995) determined individual ERD/ERS values at theoretical 46 sites of augmented 10-20 system. These electrodes were displaced over a 3-D head model approximating each "realistic" individual head model. This template model was based on the magnetic resonance data of 152 subjects, digitized at Brain Imaging Center of the Montreal Neurological Institute (SPM96).

\section{Measurement of ERD/ERS latency and amplitude}

Latency of the ERD/ERS peaks was measured for the theta, alpha 1, alpha 2, alpha 3 and gamma with respect to the zero time, taken as the onset of the cue stimulus. The ERD and ERS peaks were independently recognized by two experimenters within frontal and parietal regions of interest (ROIs). These ROIs included (i) F5, F3 and F1 electrodes for the left frontal region or FL, (ii) F6, F4 and F2 electrodes for the right frontal region or FR, (iii) P5, P3 and P1 electrodes for the left parietal region or PL and (iv) P6, P4 and $\mathrm{P} 2$ electrodes for the right parietal region or PR.

The ERD/ERS amplitude was automatically measured for each experimental condition at each of the 46 electrodes. The ROI having the maximal ERD/ERS values was considered as a reference for the latency of the topographical mapping.

\section{Statistical analysis}

Statistical comparisons were performed by ANOVA for repeated measures. Mauchley's test evaluated the sphericity assumption and the correction of the degrees of freedom was made by Greenhouse-Geisser procedure. Duncan test was used for post hoc comparisons $(P<0.05)$.

For the evaluation of the type of errors, the ANOVA comprised factors Condition (ENC, RET) and Error type (Wrong responses, Anticipated response respect to the go stimulus, Delayed response exceeding $2.5 \mathrm{~s}$ after the go stimulus). 
ENCODING AND RETRIEVAL TASKS

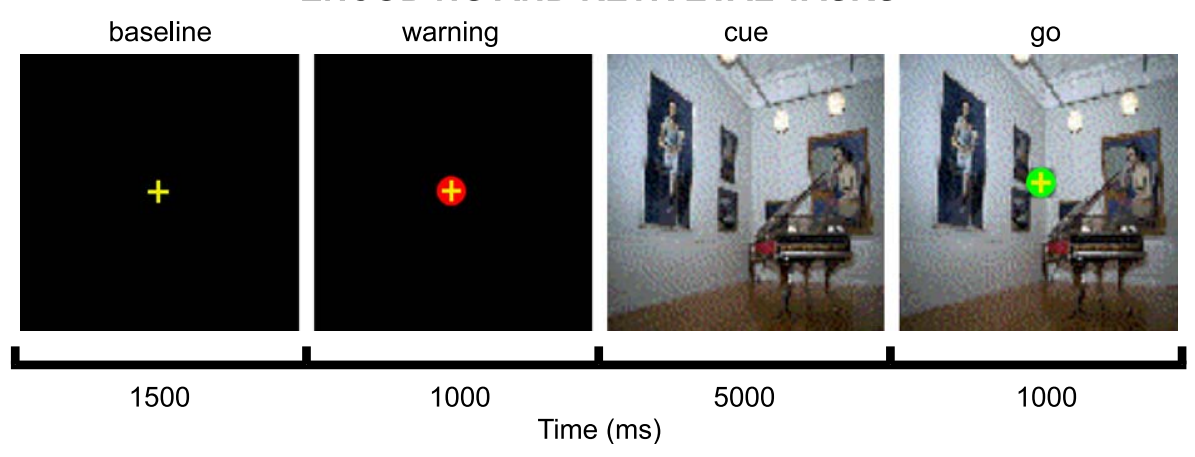

Fig. 1. Set up and experimental tasks. The experiment included an "encoding" (ENC) and a "retrieval" (RET) tasks in which 50 complex colored magazine pictures were shown after a red central target given as a visual warning stimulus (presentation time of $1 \mathrm{~s}$ ). In the ENC condition, 25 figures representing interiors of apartments ("indoor") were randomly intermingled with 25 figures representing landscapes ("landscapes"). The figures were shown one-by-one (cue period, presentation time of $5 \mathrm{~s}$ ). Subjects were instructed to press with right index finger one of the two close buttons (left $=$ "indoor"; right = "landscapes") as quickly as possible after the appearance of a green central target at the center of the figure (go stimulus, presentation time of $1 \mathrm{~s}$ ). About 1 h later, the RET condition started. In this phase, 25 figures representing previously presented "indoor" ("tests") were randomly intermingled with 25 figures representing novel "indoor" ("distractors"). Subjects were asked to discriminate between "tests" among "distractors" by pressing one of the two buttons (left $=$ "tests"; right $=$ "distractors") immediately after the go stimulus. The timing of warning stimulus, cue stimulus (picture presentation) and go stimulus was as in the "encoding" phase.

For the evaluation of movement reaction time, only the factor Condition (ENC, RET) was used on the correct hits.

For the evaluation of the ERD/ERS latency, the ANOVA analysis used the factors Condition (ENC, RET) and Time (peaks).

For the evaluation of the ERD/ERS amplitude, the ERD/ERS value of the responsive electrodes belonging to the same ROI was averaged. The mean ERD/ERS value served as an input for five ANOVA analyses for repeated measures, namely, one ANOVA for each band of interest (theta, alpha 1, alpha 2, alpha 3, gamma). The ANOVA factors were Condition (ENC, RET), ROI (FL, FR, PL, $\mathrm{PR}$ ) and Time period (at waveform peaks as revealed by the visual inspection). These ANOVA designs tested the effects of cortical
THETA

F1

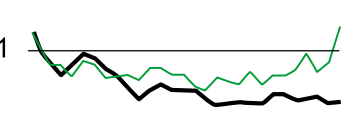

F2

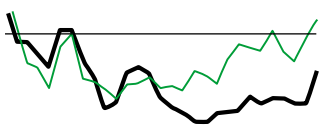

P1

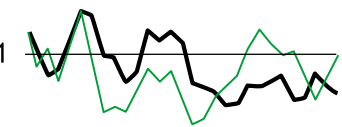

P2

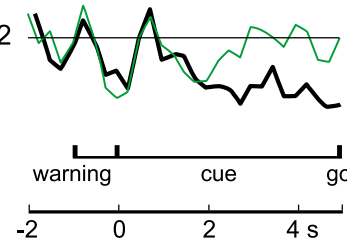

\section{ERD/ERS}

ALPHA 3

F1
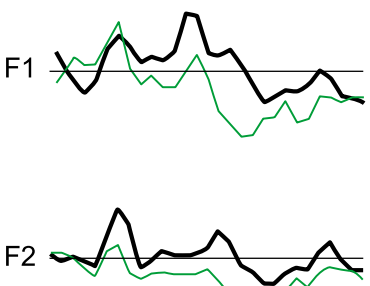

F2
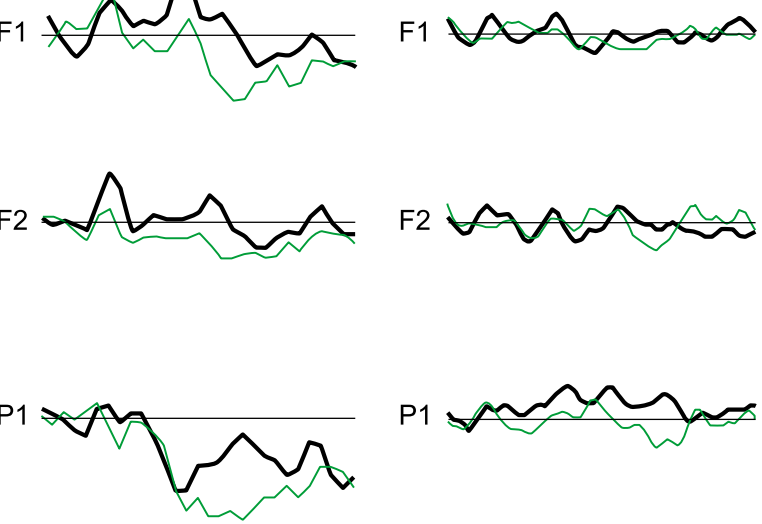

P2
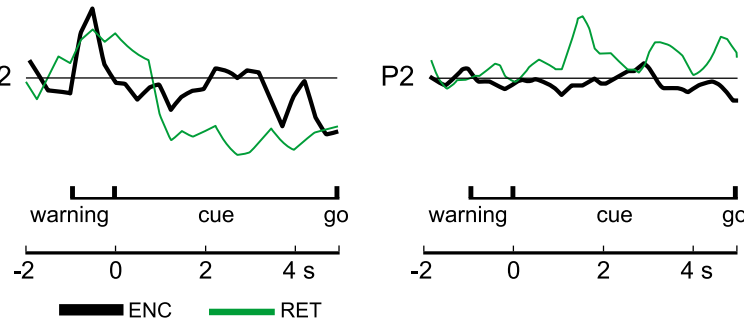

Fig. 3. The grand average waveforms of the theta, alpha 1 and gamma ERD/ERS (event-related desynchronization/synchronization) during the ENC and RET conditions. These waveforms refer to representative frontal and parietal electrode sites. 


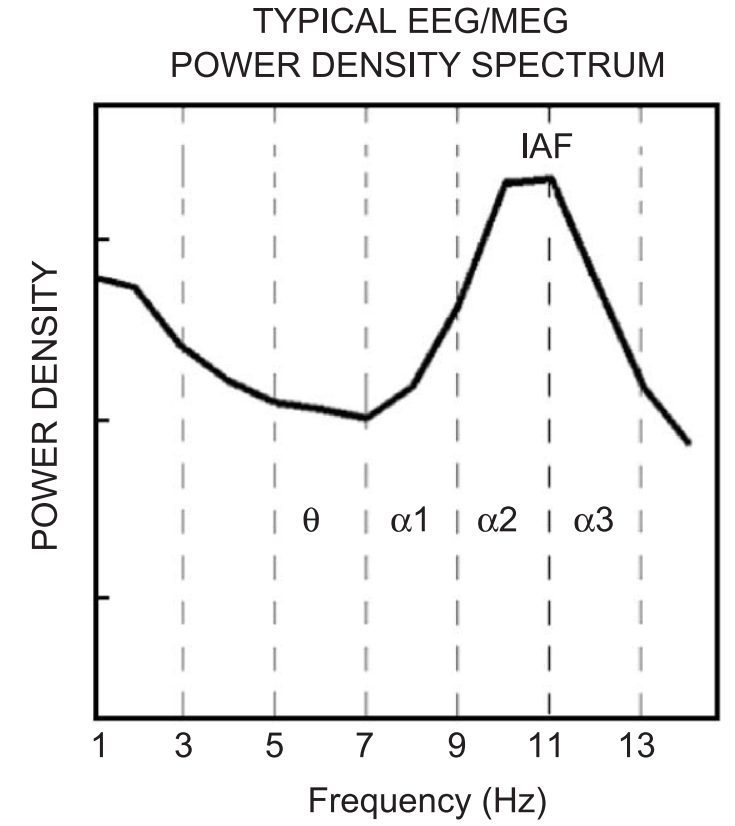

Fig. 2. Model of a typical frequency spectrum computed from EEG [or magnetoencephalographic (MEG)] data. Frequency bands of interest were theta, alpha 1 , alpha 2 and alpha 3 , which were computed according to a well-known procedure based on the detection of individual alpha frequency (IAF) peak (see Materials and methods for further explanations).

rhythmicity (ERD/ERS) on the hemispheric functional asymmetry predicted by HERA model.

\section{Results}

\section{Behavioral results}

The ANOVA analysis for the evaluation of the type of errors pointed to a statistical interaction $[F(2,18)=27.2 ; P<0.00001]$ between Condition (ENC, RET) and Error Type (Wrong responses, Anticipated response, Delayed response). Duncan post hoc testing

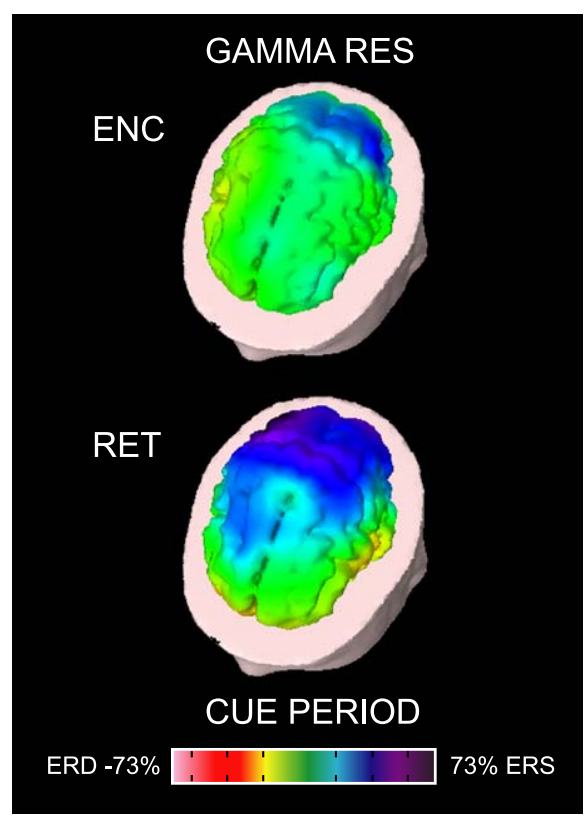

Fig. 5. Three-dimensional color maps of group gamma ERS peak during the delay period for the ENC and RET conditions. Color scale as in Fig. 4. The maximal (\%) value of the ERD/ERS is reported.

indicated that the Anticipated responses were higher for the RET than ENC condition $(P<0.00003)$.

There was no statistical difference in mean reaction time between conditions $[F(1,9)=2.49 ; P=0.15]$, namely, $696 \mathrm{~ms}( \pm 31$ $\mathrm{SE})$ and $734 \mathrm{~ms}( \pm 34 \mathrm{SE})$ for the ENC and RET tasks, respectively.

\section{Temporal evolution of ERD/“ERS” peaks}

Fig. 3 shows the group ERD/ERS waveforms of the theta, alpha 3 and gamma for the ENC and RET conditions. These waveforms refer to representative frontal and parietal electrodes. The waveforms of alpha 1 and alpha 2 are similar to alpha 3 waveform in terms of temporal evolution, according to the well-known sequence of phase-locked and nonphase-locked reactions to external stimuli (Klimesch, 1999), and for this reason are nonrepresented in this

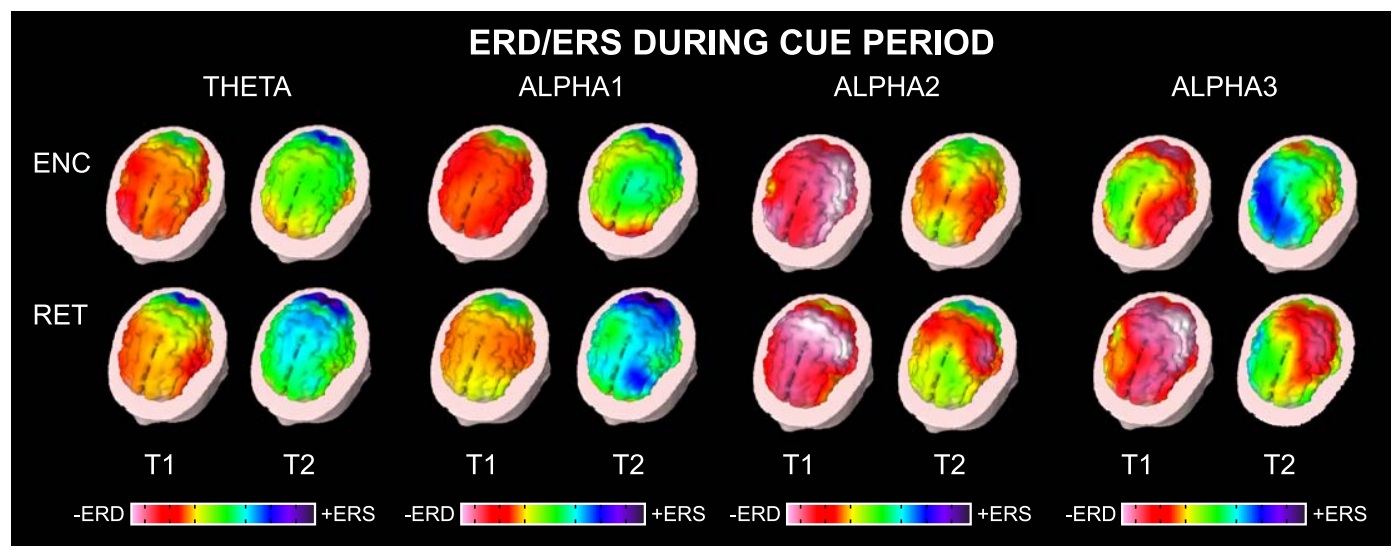

Fig. 4. Three-dimensional color maps of group theta, alpha 1, alpha 2 and alpha 3 ERD peak (T1) and ERS peak (T2) during the cue period of the ENC and RET conditions. Color scale: maximum ERD and ERS are coded in white and violet, respectively. The maximal ERD/ERS values were $\pm 65 \%$ for the theta, $\pm 64 \%$ for the alpha $1, \pm 58 \%$ for the alpha 2 and $\pm 54 \%$ for the alpha 3 . 
Table 2

Mean latency $( \pm \mathrm{SE})$ of theta, alpha 1, alpha 2, alpha 3 and gamma eventrelated desynchronization/synchronization (ERD/ERS) peaks

\begin{tabular}{|c|c|c|}
\hline & $\mathrm{T} 1$ & $\mathrm{~T} 2$ \\
\hline & mean $( \pm \mathrm{SE})$ & mean $( \pm \mathrm{SE})$ \\
\hline \multicolumn{3}{|l|}{$E N C$} \\
\hline theta & $2325( \pm 258)$ & $3650( \pm 230)$ \\
\hline alpha1 & $2625( \pm 277)$ & $3950( \pm 302)$ \\
\hline alpha2 & $2775( \pm 344)$ & $4325( \pm 201)$ \\
\hline alpha3 & $2450( \pm 263)$ & $3700( \pm 258)$ \\
\hline gamma & $1708( \pm 315)$ & - \\
\hline \multicolumn{3}{|l|}{ RET } \\
\hline theta & $1825( \pm 286)$ & $3475( \pm 222)$ \\
\hline alpha1 & $1850( \pm 167)$ & $3750( \pm 204)$ \\
\hline alpha2 & $2025( \pm 292)$ & $4275( \pm 195)$ \\
\hline alpha3 & $1925( \pm 281)$ & $3425( \pm 396)$ \\
\hline gamma & $1361( \pm 141)$ & - \\
\hline
\end{tabular}

These peaks refer to both encoding (ENC) and retrieval (RET) conditions. Zero time indicates the onset of the visual cue stimulus (i.e., complex colored magazine picture).

figure. During the period of interest (cue period), the waveforms of theta and alpha (sub-bands) showed an "early" ERS peak close to beginning of the figure presentation. It was first followed by an ERD peak (T1) and then by a reduced ERD that in some cases could become a "late" ERS peak (T2) (i.e., for sake of brevity, we called "ERS" peaks both reduced ERD and true ERS). Reasonably, the early ERS represented the phase-locked EEG oscillations evoked or induced by the visual warning stimulus and was not further considered. Instead, the gamma waveforms showed a parietal ERS peak (T1) after the end of evoked potentials.

Table 2 reports the mean $( \pm S E)$ latency of the theta, alpha and gamma ERD/ERS during the cue period. The only statistical difference was that the theta and alpha ERD/ERS peak was later during the ENC than RET condition [main effect Condition, $F(1,9)=5.77 ; P<0.04]$.

\section{Spatial distribution of ERD/ERS peaks}

Figs. 4 and 5 illustrate the topographical maps of the theta, alpha and gamma ERD/“ERS" peaks during the cue period. For the ENC condition, the theta and alpha 1 ERD peak (time1, T1) was distributed over bilateral central and frontal areas, while alpha 2 and alpha 3 ERD peak (T1) was mainly represented in the left centroparietal areas. Afterward its peak, the ERD amplitude markedly reduced over the whole scalp up to the "ERS" peak (time 2, T2). The maps for the RET condition were principally characterized by a stronger alpha 3 ERD peak (T1) in left centroparietal area and a less reduction of the alpha 3 ERD at T2. Furthermore, the gamma ERS peak was represented in left parietal area for the ENC condition and in bilateral parietal area (stronger in right than left parietal areas) for the RET condition.

\section{Statistical analysis of ERD/"ERS"}

Based on the visual inspection of the waveforms during the cue period, the ANOVA designs for theta, alpha 1, alpha 2 and alpha 3 included a factor Time (ERD peak as T1 and ERS peak as T2). Instead, the ANOVA design for gamma had no factor Time since only an ERS peak was recognized. For all bands of interest, the other factors were Condition (ENC, RET) and ROI (FL, FR, PL, PR).

The ANOVA for theta ERD/ERS amplitude pointed to a main statistical effect $[F(1,9)=7.277 ; \mathrm{MSe}=1,465 ; P<0.02]$ for Condition, indicating a lesser ERD and a stronger ERS for the RET than ENC conditions.

Analogously to theta, the ANOVA for alpha 1 showed a main statistical effect $[F(1,9)=17.57$; MSe $=1,118 ; P<0.002]$ for Condition, indicating a lesser ERD and a stronger ERS for the RET than ENC conditions.

No ANOVA statistical significance was obtained for alpha 2.

The ANOVA for alpha 3 showed a main statistical effect $[F(1,9)=5.18 ; \mathrm{MSe}=3,664 ; P<0.04]$ for Condition, indicating a greater ERD and a lesser ERS for the RET than ENC conditions. On the whole, the ANOVA for theta, alpha 1, alpha 2 and alpha 3 showed no evidence in line with HERA model.

On the contrary, the ANOVA for gamma indicated a statistical interaction $[F(3,24)=7.873$; $\mathrm{MSe}=774 ; P<0.0008]$ between Condition (ENC, RET) and ROI (FL, FR, PL, PR). Fig. 6 illustrates the means $( \pm \mathrm{SE})$ of the ERS representing these statistical results. Duncan post hoc indicated that the ERS peak was stronger for RET than ENC conditions in right parietal area $(P<$

\section{GAMMA AMPLITUDE AT T1 FOR TASK AND ROI}
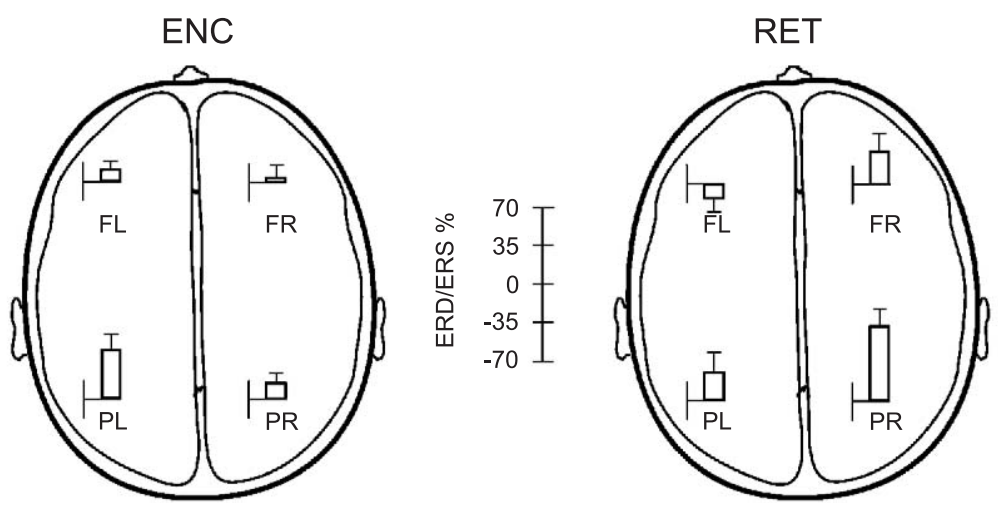

Fig. 6. Across-subject means ( $\pm \mathrm{SE}$ ) of the gamma ERD/ERS amplitudes as provided by the ANOVA design. In particular, these means refer to a statistical interaction between the factors Condition (ENC, RET) and ROI (FL, FR, PL, PR). 
0.0009). Furthermore, for ENC condition, the ERS peak was stronger in left than right parietal areas $(P<0.04)$, while, for RET condition, ERS peak was stronger in right than left frontal areas $(P<0.008)$ and in right than left parietal areas $(P<0.005)$. These results thus indicate the presence of an asymmetry in agreement with the HERA model in its most recent and stringent formulation (Habib et al., 2003), which however involves the parietal, rather than the prefrontal areas.

Of note, a control ANOVA design for each band of interest demonstrated that the above findings were not due to a different baseline band power.

\section{Discussion}

\section{Methodological remarks}

In the present study, brain rhythms accompanying the visuospatial long-term episodic memory task were investigated by the topographical mapping of the theta, alpha and gamma ERD/ERS. As an advantage, the mapping procedure required no computational assumption such as the number of equivalent dipoles or the regularization parameters of the linear inverse estimation (Babiloni et al., 2001a). In previous studies, the mapping of the ERD/ERS has provided very interesting findings on brain rhythms accompanying somatosensory, motor and cognitive demands (Babiloni et al., 1999, 2000, 2001b, 2002; Gevins and Smith, 2000; Gevins et al., 1998; McEvoy et al., 2001; Okada and Salenius, 1998). As a limitation, the cortical sources of the ERD/ERS must be inferred with caution based on the topographical mapping. Indeed, surface Laplacian maxima could not precisely overlie the corresponding cortical sources due to the influence of tangentially oriented generators (Babiloni et al., 1995, 1996). Here, we accounted for such a limitation considering relatively wide frontal and parietal regions of interest to model the activity of underlying prefrontal and posterior parietal areas.

\section{Theta and alpha ERD/ERS do not fit the HERA model}

Compared to the ENC condition, the RET condition was characterized by a posterior theta ERS $(4-6 \mathrm{~Hz})$ and a stronger high alpha $(10-12 \mathrm{~Hz})$ ERD followed by a lower rebound (alpha ERS). These results are globally in line with previous evidence (Klimesch et al., 1996, 1997, 1999) showing that theta and upper alpha rhythms are sensitive to semantic long-term memory processes and retrieval processes, possibly as a reflection of the gating oscillatory activity within thalamocortical (alpha) and hippocampocortical (theta) feedback loops. Noteworthy, the present results did not show any asymmetry in the theta and alpha rhythms compatible with the HERA model. This is in line with previous studies showing that theta and alpha rhythms are widely distributed rhythms over both hemispheres, especially with engaging cognitive demands involving working memory, focused attention and semantic categorization (Klimesch et al., 1996, 1997, 1999; Pfurtscheller et al., 1997; Pfurtscheller and Klimesch, 1992).

\section{HERA-like asymmetric posterior gamma ERS}

In the present study, the gamma responses (ERS) were prominent in the posterior scalp areas. The ENC condition induced a stronger gamma ERS in left than right parietal area, while the RET condition showed a prominent gamma ERS in right compared to left parietal area. This hemispheric asymmetry did not regard the frontal responses and, therefore, seems to be inconsistent with the classical formulation of the HERA predictions, which is based on the function of the prefrontal areas (Habib et al., 2003). Furthermore, an evident right frontal gamma ERS was observed in RET condition but not in ENC condition.

A parsimonious explanation of the present results is that gamma rhythm was principally related to the synchronization or "temporal binding" of the parietal neuronal populations for the processing of visuospatial inputs, regardless the specific processes of ENC and RET. Indeed, it has been shown that visuospatial demands modulate gamma and alpha oscillations especially in the parietaloccipital regions (Dujardin et al., 1994, 1995; Gonzalez-Hernandez et al., 2002; Tallon-Baudry et al., 1999). Furthermore, it has been suggested that gamma band oscillations mainly reflect the elaboration of complex sensory stimuli over primary and nonprimary sensory areas and may bind the cooperative processing of nodes with a brain neural network (Engel and Singer, 2001; Keil et al., 1999; Rodriguez et al., 1999; Tallon-Baudry et al., 1997, 1999). Finally, it has been demonstrated that gamma oscillations over lateral and medial parietal cortices mediate the interaction of the coherent visual representation with the experience-based perception of objects (Engel and Singer, 2001; Keil et al., 1999; Rodriguez et al., 1999; Tallon-Baudry et al., 1997).

As a crucial limitation, the above explanation does not account for the different topography of the gamma responses during the ENC and RET conditions, despite the similar features of the visual images used in those conditions. An alternative explanation is that the present parietal gamma responses may probe the sensoryperceptual processing facet of the ENC and RET processes. During the ENC phase, the left parietal gamma responses would reflect the cortical representation of the visual event. Whereas during the RET phase, the right parietal gamma responses would match the actual visuospatial stimuli with the sensory representations formed by the ENC processes. This stage of processing would represent a sort of HERA-like sensory mode of neuronal synchronization. The "sensory" nature of the gamma responses would explain why the gamma responses were prominent in the parietal respect to the frontal cortical areas.

The present gamma responses complement previous neuroimaging data showing the putative REMO cerebral sites at frontal and anterior cingulate areas (Lepage et al., 2000; Nyberg et al., 1996; Tulving et al., 1994). This kind of activation is also confirmed by recent studies (Rossi et al., 2001; Sandrini et al., 2004), showing that the rTMS of right DLPFC mainly impaired the retrieval of the encoded visual information. The lack of gamma synchronization on left frontal areas during encoding could be due to a minor cognitive demand requested in the encoding process as compared to the retrieval phase. On the contrary, the retrieval process needs a full activation of right frontoparietal network. Gamma band synchronization seems to support this higher cognitive demand, binding frontal and parietal areas in an integrated activation (Lutzenberger et al., 2002; Niedermayer, 2003; Tallon-Baudry et al., 1998).

The fact that frontal gamma synchronization did not fit HERA model gives rise to the importance of a multimodal neuroimaging approach. Combining different neurophysiological techniques (i.e., exploring brain activity from different perspectives) during successful episodic long-term memory may therefore contribute to better address the parallel frontoparietal processes at the basis of cortical functional asymmetries. As a matter of fact, the idea of a 
unique pattern of "cortical activation/deactivation" explaining long-term memory processes seems too restrictive and moreover too much dependent by the technique utilized to investigate brain functions. An alternative view might be that a comprehensive model of cognitive brainwork should take into account several indexes of parallel functional processing, including brain rhythmicity, hemodynamic and blood flow responses and rTMS interference to investigate the cognitive challenge, according to a true multidimensional approach to the working brain.

\section{Conclusion}

This high-resolution EEG study in young adults showed the frontoparietal theta, alpha and gamma ERD/ERS during a visuospatial long-term episodic memory task including ENC and RET phases. The aim was to disclose whether the brain rhythmicity presented a pattern compatible with the predictions of the HERA model. The ENC phase was associated with prominent gamma responses over left parietal cortex, possibly contributing to form long-lasting representation of the sensory stimuli. The RET phase was associated with prominent gamma responses over right parietal cortex, possibly contributing for a matching of visual inputs with sensory representations formed during the ENC phase. The fact that frontal gamma synchronization did not fit HERA model gives rise to the importance of a multimodal neuroimaging approach in which different techniques could disclose the different facets of the physiological mechanisms subserving long-term episodic memory.

\section{References}

Babiloni, F., Babiloni, C., Fattorini, L., Carducci, F., Onorati, P., Urbano, A., 1995. Performances of surface Laplacian estimators: a study of simulated and real scalp potential distributions. Brain Topogr. 8 (1), $35-45$.

Babiloni, F., Babiloni, C., Carducci, F., Fattorini, L., Onorati, P., Urbano, A., 1996. Spline Laplacian estimate of EEG potentials over a realistic magnetic resonance-constructed scalp surface model. Electroencephalogr. Clin. Neurophysiol. 98 (4), 363-373.

Babiloni, F., Carducci, F., Babiloni, C., Urbano, A., 1998. Improved realistic Laplacian estimate of highly-sampled EEG potentials by regularization techniques. Electroencephalogr. Clin. Neurophysiol. 106 (4), $336-343$.

Babiloni, C., Carducci, F., Cincotti, F., Rossini, P.M., Neuper, C., Pfurtscheller, G., Babiloni, F., 1999. Human movement-related potentials vs. desynchronization of EEG alpha rhythm: a high-resolution EEG study. Neuroimage 10 (6), 658-665.

Babiloni, C., Babiloni, F., Carducci, F., Cincotti, F., Del Percio, C., De Pino, G., Maestrini, S., Priori, A., Tisei, P., Zanetti, O., Rossini, P.M., 2000. Movement-related electroencephalographic reactivity in Alzheimer disease. Neuroimage 12 (2), 139-146.

Babiloni, F., Carducci, F., Cincotti, F., Del Gratta, C., Pizzella, V., Romani, G.L., Rossini, P.M., Tecchio, F., Babiloni, C., 2001a. Linear inverse source estimate of combined EEG and MEG data related to voluntary movements. Hum. Brain Mapp. 14 (4), 197-209.

Babiloni, C., Babiloni, F., Carducci, F., Cincotti, F., Rosciarelli, F., Rossini, P., Arendt-Nielsen, L., Chen, A., 2001b. Mapping of early and late human somatosensory evoked brain potentials to phasic galvanic painful stimulation. Hum. Brain Mapp. 12 (3), 168-179.

Babiloni, C., Babiloni, F., Carducci, F., Cincotti, F., Rosciarelli, F., ArendtNielsen, L., Chen, A.C., Rossini, P.M., 2002. Human brain oscillatory activity phase-locked to painful electrical stimulations: a multi-channel EEG study. Hum. Brain Mapp. 15 (2), 112-123.
Brewer, J.B., Zhao, Z., Desmond, J.E., Glover, G.H., Gabrieli, J.D., 1998. Making memories: brain activity that predicts how well visual experience will be remembered. Science 281 (5380), 1185-1187.

Buckner, R.L., Wheeler, M.E., 2000. The cognitive neuroscience of remembering. Nat. Rev., Neurosci. 2 (9), 624-634.

Buckner, R.L., Kelley, W.M., Petersen, S.E., 1999. Frontal cortex contributes to human memory formation. Nat. Neurosci. 12 (4), 311-314.

Dujardin, K., Bourriez, J.L., Guieu, J.D., 1994. Event-related desynchronization (ERD) patterns during verbal memory tasks: effect of age. Int. J. Psychophysiol. 16 (1), 17-27.

Dujardin, K., Bourriez, J.L., Guieu, J.D., 1995. Event-related desynchronization (ERD) patterns during memory processes: effects of aging and task difficulty. Electroencephalogr. Clin. Neurophysiol. 96 (2), $169-182$.

Engel, A.K., Singer, W., 2001. Temporal binding and the neural correlates of sensory awareness. Trends Cogn. Sci. 5 (1), 16-25.

Fletcher, P.C., Henson, R.N.A., 2001. Frontal lobes and human memory. Insights from functional neuroimaging. Brain 124, 849-881.

Gevins, A., Smith, M.E., 2000. Neurophysiological measures of working memory and individual differences in cognitive ability and cognitive style. Cereb. Cortex 10 (9), 829-839.

Gevins, A., Smith, M.E., Leong, H., McEvoy, L., Whitfield, S., Du, R., Rush, G., 1998. Monitoring working memory load during computerbased tasks with EEG pattern recognition methods. Hum. Factors 40 (1), 79-91.

Gonzalez-Hernandez, J.A., Pita-Alcorta, C., Cedeno, I., Bosch-Bayard, J., Galan-Garcia, L., Scherbaum, W.A., Figueredo-Rodriguez, P., 2002. Wisconsin Card Sorting Test synchronizes the prefrontal, temporal and posterior association cortex in different frequency ranges and extensions. Hum. Brain Mapp. 17 (1), 37-47.

Habib, R., Nyberg, L., Tulving, E., 2003. Hemispheric asymmetries of memory: the HERA model revisited. Trends Cogn. Sci. 7 (6), 241-245.

Hallett, M., 2000. Trascranial magnetic stimulation and the human brain. Nature 406, 147-150.

Keil, A., Muller, M.M., Ray, W.J., Gruber, T., Elbert, T., 1999. Human gamma band activity and perception of a gestalt. J. Neurosci. 19 (16), $7152-7161$.

Kirchhoff, B.A., Wagner, A.D., Maril, A., Stern, C.E., 2000. Prefrontaltemporal circuitry for episodic encoding and subsequent memory. J. Neurosci. 20, 6173-6180.

Klimesch, W., 1996. Memory processes, brain oscillations and EEG synchronization. Int. J. Psychophysiol. 24 (1-2), 61-100.

Klimesch, W., 1999. EEG alpha and theta oscillations reflect cognitive and memory performance: a review and analysis. Brain Res. Brain Res. Rev. $29(2-3), 169-195$.

Klimesch, W., Doppelmayr, M., Russegger, H., Pachinger, T., 1996. Theta band power in the human scalp EEG and the encoding of new information. NeuroReport 7 (7), 1235-1240.

Klimesch, W., Doppelmayr, M., Pachinger, T., Russegger, H., 1997. Event-related desynchronization in the alpha band and the processing of semantic information. Brain Res. Cogn. Brain Res. 6 (2), 83-94.

Klimesch, W., Doppelmayr, M., Russegger, H., Pachinger, T., Schwaiger, J., 1998. Induced alpha band power changes in the human EEG and attention. Neurosci. Lett. 244 (2), 73-76.

Klimesch, W., Doppelmayr, M., Schwaiger, J., Auinger, P., Winkler, T., 1999. Related 'Paradoxical' alpha synchronization in a memory task. Brain Res. Cogn. Brain Res. 7 (4), $493-501$.

Lepage, M., Ghaffar, O., Nyberg, L., Tulving, E., 2000. Prefrontal cortex and episodic memory retrieval mode. Proc. Natl. Acad. Sci. 97, $506-511$.

Lutzenberger, W., Ripper, B., Busse, L., Birbaumer, N., Kaiser, J., 2002. Dynamics of gamma-band activity during an audiospatial working memory task in humans. J. Neurosci. 22 (13), 5630-5638.

McEvoy, L.K., Pellouchoud, E., Smith, M.E., Gevins, A., 2001. Neurophysiological signals of working memory in normal aging. Brain Res. Cogn. Brain Res. 11 (3), 363-376. 
Moretti, D.V., Babiloni, F., Carducci, F., Cincotti, F., Remondini, E., Rossini, P.M., Salinari, S., Babiloni, C., 2003. Computerized processing of EEG-EOG-EMG artifacts for multi-centric studies in EEG oscillations and event-related potentials. Int. J. Psychophysiol. 47 (3), $199-216$

Niedermeyer, E., 2003. Electrophysiology of the frontal lobe. Clin. Electroencephalogr. 34, 5-12.

Nolde, S.F., Johnson, M.K., D’Esposito, M., 1998. Left prefrontal activation during episodic remembering: an event-related fMRI study. NeuroReport 9 (15), 3509-3514.

Nunez, P., 1995. Neocortical Dynamics and Human EEG Rhythms. Oxford Univ. Press, New York.

Nyberg, L., McIntosh, A.R., Cabeza, R., Habib, R., Houle, S., Tulving, E., 1996. General and specific brain regions involved in encoding and retrieval of events: what, where, and when. Proc. Natl. Acad. Sci. U. S. A. 93 (20), 11280-11285.

Okada, Y.C., Salenius, S., 1998. Roles of attention, memory and motor preparation in modulating human brain activity in a spatial working memory task. Cereb. Cortex 8 (1), 80-96.

Pascual-Leone, A., Walsh, V., Rothwell, J., 2000. Transcranial magnetic stimulation in cognitive neuroscience-Virtual lesion, chronometry, and functional connectivity. Curr. Opin. Neurobiol. 10, 232-237.

Pfurtscheller, G., Aranibar, A., 1979. Evaluation of event-related desynchronization (ERD) preceding and following voluntary self-paced movement. Electroencephalogr. Clin. Neurophysiol. 46, 138-146.

Pfurtscheller, G., Klimesch, W., 1992. Functional topography during a visuoverbal judgment task studied with event-related desynchronization mapping. J. Clin. Neurophysiol. 9, 120-131.

Pfurtscheller, G., Neuper, C., 1994. Event-related synchronization of mu rhythm in the EEG over the cortical hand area in man. Neurosci. Lett. 174 (1), 93-96

Pfurtscheller, G., Neuper, C., Flotzinger, D., Pregenzer, M., 1997. EEGbased discrimination between imagination of right and left hand movement. Electroencephalogr. Clin. Neurophysiol. 103 (6), 642-651.
Ranganath, C., Johnson, M.K., D’Esposito, M., 2000. Left anterior prefrontal activation increases with demands to recall specific perceptual information. J. Neurosci. 20 (22), RC108.

Rodriguez, E., George, N., Lachaux, J.P., Martinerie, J., Renault, B., Varela, F.J., 1999. Perception's shadow: long-distance synchronization of human brain activity. Nature 397 (6718), 430-433.

Rossi, S., Cappa, S.F., Babiloni, C., Pasqualetti, P., Miniussi, C., Carducci, F., Babiloni, F., Rossini, P.M., 2001. Prefrontal cortex in long-term memory: an "interference" approach using magnetic stimulation. Nat. Neurosci. 4 (9), 948-952.

Rossini, P.M., Rossi, S., 1998. Clinical applications of motor evoked potentials. Electroencephalogr. Clin. Neurophysiol. 106 (3), 180-194.

Sandrini, M., Cappa, S.F., Rossi, S., Rossini, P.M., Miniussi, C., 2004. The role of prefrontal cortex in a verbal memory task. J. Cogn. Neurosci. 15 (6), $1-7$.

Tallon-Baudry, C., Bertrand, O., Delpuech, C., Permier, J., 1997. Oscillatory gamma-band $(30-70 \mathrm{~Hz})$ activity induced by a visual search task in humans. J. Neurosci. 17 (2), $722-734$.

Tallon-Baudry, C., Bertrand, O., Peronnet, F., Pernier, J., 1998. Induced gamma-band activity during the delay of a visual short-term memory task in humans. J. Neurosci. 18, 4244-4254.

Tallon-Baudry, C., Kreiter, A., Bertrand, O., 1999. Sustained and transient oscillatory responses in the gamma and beta bands in a visual short-term memory task in humans. Vis. Neurosci. 16 (3), 449-459.

Tulving, E., Kapur, S., Craik, F.I., Moscovitch, M., Houle, S., 1994. Hemispheric encoding/retrieval asymmetry in episodic memory: positron emission tomography findings. Proc. Natl. Acad. Sci. U. S. A. 91, 2016-2020.

Wagner, A.D., Koutstaal, W., Schacter, D.L., 1999. Related articles, links when encoding yields remembering: insights from event-related neuroimaging. Philos. Trans. R. Soc. London, Ser. B 354 (1387), $1307-1324$.

Walsh, V., Cowey, A., 2000. Transcranial magnetic stimulation and cognitive neuroscience. Nat. Rev., Neurosci. 1, 73-79. 